\title{
Matrix bands - their use and sterilisation in general practice
}

\author{
A survey of the use of matrix bands and their decontamination in general dental practice
}

\author{
A. H. Lowe, F. J. T. Burke, S. McHugh and J. Bagg. Br Dent J 2002; 192: 40-42
}

\begin{abstract}
Aims
The aims of this study were to determine the pattern of use and re-use of matrix bands in general practice in Scotland, to demonstrate which type of matrix band is most commonly used and to examine infection control measures of relevance to the safe use and re-use of matrix bands.
\end{abstract}

Materials and methods

Subjects: 621 of Scotland's 1,849 general dental practitioners were randomly selected. Data collection: A 19-item self-reported questionnaire was mailed in June 1999 with a follow-up mailing sent in August 1999. Analysis: Data analysis involved descriptive statistics and cross-tabulation. Where appropriate, differences between categories were tested for significance by a Chi-square test.

\section{Results}

A total of 479 questionnaires were returned, representing a response rate of $77 \%$. Reported compliance with routine glove wearing was high (91\%). Most dentists (92\%) provided training on instrument cleaning and sterilisation for their dental nurses. Ultrasonic baths were used by $59 \%$ of practitioners; the remainder soaked or manually scrubbed instruments to remove debris before autoclaving. The Siqveland matrix was the matrix of choice for $96 \%$ of respondents. $7 \%$ provided a new matrix band for each patient. Most (64\%) changed bands only when they were bent or damaged; $29 \%$ changed them daily or weekly. Deterrents to use of a new band for each patient were cost (39\%) and time (52\%). A total of 54\% of respondents considered matrix band replacement unnecessary between patients.

\section{Conclusions}

The Siqveland matrix band is the most popular among the study group of dental practitioners. Re-use of matrix bands is common. Guidelines for the safe re-use of matrix bands are required.

\section{IN BRIEF}

- The Siqveland matrix is by far the most popular in general dental practice in Scotland.

- Most dental practitioners do not remove and change the matrix band between patients, relying on routine instrument decontamination procedures.

- Time, cost and lack of perception of need were the main barriers identified to changing bands between patients.

- Most dentists who reponded had provided their dental nurses with training in cleaning and sterilisation of instruments.

- Hand-scrubbing remains a component of instrument cleaning protocols in many dental surgeries, but ultrasonic baths were used by $59 \%$ of practitioners.

\section{COMMENT}

Concern about control of cross-infection tends to go through peaks and troughs according to the perceived risks from the latest infective scare. HIV and Hepatitis B have stimulated improvements to practice and presently there is interest in the causative agent of new variant Creuzfeld-Jacob disease. Whether and to what extent this agent is transmissible by operative dental procedures is still unknown but it seems timely to look again at our cross-infection control procedures. This paper addresses an issue that has not received adequate attention in the past, namely the use and decontamination of matrix bands. These usually contact the gingiva during use, often becoming contaminated with blood but there is little guidance about the best way to prevent cross-infection from matrix bands. For that reason the authors have tried to obtain 'base line' information on how GDPs presently deal with the problem.

The data presented was self-reported by approximately one third of GDPs in Scotland using a questionnaire. Almost all practitioners (96\%) used the Siqveland matrix band. Only $7 \%$ of respondents used a new matrix band for each patient, while the majority did not consider them to be single use disposable items and generally only replaced them when damaged. Interestingly $30 \%$ of GDPs changed bands at daily or weekly intervals but it is not clear why this period was chosen. There seems little point in routinely discarding bands after one week, the risks of transmission of infection are no less than after two or three weeks use. It would seem more logical to either dispose of matrix bands after each patient or else adequately clean and sterilise them after each use but continue to use them until they lose their mechanical efficiency.

Most practices decontaminated matrix bands between patients although the details of the regime used varied between practices. It is encouraging to see that $99 \%$ of practices used autoclaving to sterilise matrix bands. However, there was less of a consensus about the pre-sterilisation cleaning. Approximately $59 \%$ used ultrasonic baths as part of the procedure and $60 \%$ used manual scrubbing either instead of ultrasonic cleaning or as a supplement to it. Three percent of practices used presoaking without scrubbing as the cleaning method, but this is not adequate for removal of organic material and without such cleaning the steam may not penetrate sufficiently to kill infectious agents. This paper, therefore, is useful in giving an overview of current practice and to reflect on our own practice. What it does not do is make suggestions for what is best practice, although recommendations are put forward in another allied paper by the same group.

\section{W. Ian Douglas}

Reader in Oral Microbiology, Department of Oral Pathology, School of Clinical Dentistry, Sheffield 